Editorial

\title{
Yeast Biotechnology 2.0
}

\author{
Ronnie G. Willaert
}

Alliance Research Group VUB-UGent NanoMicrobiology (NAMI), IJRG VUB-EPFL NanoBiotechnology \& NanoMedicine (NANO), Research Group Structural Biology Brussels, Vrije Universiteit Brussel, 1050 Brussels, Belgium; Ronnie.Willaert@vub.be; Tel.: +32-26291846

Received: 31 October 2018; Accepted: 22 November 2018; Published: 23 November 2018

check for updates

Keywords: Saccharomyces cerevisiae; non-Saccharomyces yeasts; fermentation-derived products; fermented beverages; wine; beer; mead; flavor; citric acid production; bioethanol production; enzyme production; bioreactors; nanobiotechnology

Yeast biotechnology. For thousands of years, yeasts have been used for the making of bread and the production of fermented alcoholic drinks, such as wine and beer. Saccharomyces cerevisiae (bakers' and brewers' yeast) is the yeast species that is surely the most exploited by man. Nowadays, Saccharomyces is a cornerstone of modern biotechnology and also a top choice organism for industrial production of fuels, chemicals, and pharmaceuticals. Today, more and more different yeast species are explored for industrial applications. This Special Issue "Yeast Biotechnology 2.0" is a continuation of the first issue "Yeast Biotechnology" (https:/ / www.mdpi.com/books/pdfview/book/324).

Yeast synthetic biology and strain engineering. Recently, important progress has been made in unlocking the key elements in the biochemical pathways involved in the synthesis of aroma compounds, as well as in methods to engineer these pathways. Recent advances in bioengineering of yeasts-including S. cerevisiae - to produce aroma compounds and bioflavors are reviewed in Reference [1]. This review presents yeast as a significant producer of bioflavors in a fresh context and proposes new directions for combining engineering and biology principles to improve the yield of targeted aroma compounds. In a proof-of-concept study, Yarrowia lipolytica was used as a whole cell factory for the de novo production of long chain dicarboxylic acid (LCDA-16 an -18) using glycerol as the sole carbon source [2]. The results provide basis for developing Y. lipolytica as a safe biorefinery platform for sustainable production of high-value LCDCAs from non-oily feedstock. It was demonstrated that a mutant strain of $Y$. lipolytica can be used to produce citric acid from renewable carbon sources such as rapeseed oil, glycerol, and glycerol-containing waste of the biodiesel industry and glucose-containing aspen waste [3]. The cost-effective production of cellulosic ethanol requires robust microorganisms for rapid co-fermentation of glucose and xylose. Therefore, a recombinant diploid xylose-fermenting S. cerevisiae strain was developed by integrating Piromyces sp. E2 xylose isomerase (PirXylA) and Orpinomyces sp. ukk1 xylose (OrpXylA) in the genome in multiple copies [4]. The development of a counter-selection method for phenyl auxotrophy could be a useful tool in the repertoire of yeast genetics. A fluorinated precursor, i.e., 4-fluorophenylpyruvate (FPP), was found to be toxic to several strains from Saccharomyces and Candida genera [5]. The results show that FPP could effectively be used for counter-selection, but not for enhanced phenylethanol production.

New developments in efficient biomolecule production. In recent years, interest in the industrial production of yeast $\beta$-glucan has increased since it is an immunostimulant molecule for human and animal health. The $\beta$-glucan yield was optimised during anaerobic fermentation by evaluating the effect of the carbon source (glucose) and $\mathrm{NaCl}$ osmotic stress [6]. A yeast isolate, selected for its lipolytic activity from a meat product, was characterized as Pichia anomala [7]. Submerged fermentation optimization resulted in a significantly increased production of an extracellular lipolytic enzyme. 
Fermented beverages: beer, wine and honey fermentation. Nowadays, wild yeasts are explored for beer and wine making to increase the natural flavor diversity of fermented beverages. Flavor was added to beer by performing mixed fermentation using non-Saccharomyces cerevisiae/pastorianus yeasts [8]. For this, a total of 60 strains belonging to the genera Candida, Pichia, and Wickerhamomyces were evaluated. Several strains produced substantially higher amounts of aroma alcohols and esters compared to a reference lager yeast strain.

Proline is the predominant amino acid in grape juice, but it is poorly assimilated by wine yeast under the anaerobic conditions of most fermentation. A novel wine yeast mutant that was obtained through ethyl methanesulfonate (EMS) mutagenesis, showed a markedly increased proline utilization and could be used to perform fermentations in nitrogen-limited conditions [9]. Icewine is a sweet dessert wine produced from grapes naturally frozen on the vine. Since acetic acid is undesired in Icewine, the yeast cytosolic redox status and its correlation to acetic acid production was investigated [10]. Yeasts involved in veil formation during the biological aging of Sherry wines are mainly S. cerevisiae, which are traditionally been divided in the varieties beticus, cheresiensis, montuliensis and rouxii. A microtiter plate assay method was developed to assure the identification and classification of veil-forming yeasts during Sherry wine aging [11].

Honey fermentations are usually performed using almost exclusively yeasts in the genus Saccharomyces. To increase the yeast biodiversity, two strains of Torulaspora delbrueckii were isolated from the gut of a locally collected honey bee [12]. These wild yeast fermentations displayed better sensory characteristics than mead fermentations by a champagne yeast, and mixed fermentations of the wild and the champagne yeast resulted in a rapid industrial fermentation process.

Yeast nanobiotechnology. Clinical needs for novel antifungal agents have increased due to the increase of people with a compromised immune system, and the appearance of resistant fungi and infections by unusual yeasts. In recent years, several micro- and nanoscale approaches have been introduced for antifungal drug discovery. These are reviewed in the last contribution to this special issue [13].

In summary, this Special Issue compiles the current state-of-the-art of research and technology in the area of "yeast biotechnology" and highlights prominent current research directions in the fields of yeast synthetic biology and strain engineering, new developments in efficient biomolecule production, fermented beverages (beer, wine, and honey fermentation), and yeast nanobiotechnology. We very much hope that you enjoy reading it and looking forward to the next special issue "Yeast Biotechnology 3.0" to appear in 2019 (https: / / www.mdpi.com/journal/fermentation/special_issues/yeast3).

Acknowledgments: The Belgian Federal Science Policy Office (Belspo) and the European Space Agency (ESA) PRODEX program supported this work. The Research Council of the Vrije Universiteit Brussel (Belgium) and the University of Ghent (Belgium) are acknowledged to support the Alliance Research Group VUB-UGhent NanoMicrobiology (NAMI), and the International Joint Research Group (IJRG) VUB-EPFL BioNanotechnology \& NanoMedicine (NANO).

Conflicts of Interest: The author declares no conflict of interest.

\section{References}

1. Van Wyk, N.; Kroukamp, H.; Pretorius, I. The Smell of Synthetic Biology: Engineering Strategies for Aroma Compound Production in Yeast. Fermentation 2018, 4, 54. [CrossRef]

2. Abghari, A.; Madzak, C.; Chen, S. Combinatorial Engineering of Yarrowia lipolytica as a Promising Cell Biorefinery Platform for the de novo Production of Multi-Purpose Long Chain Dicarboxylic Acids. Fermentation 2017, 3, 40. [CrossRef]

3. Morgunov, I.; Kamzolova, S.; Lunina, J. Citric Acid Production by Yarrowia lipolytica Yeast on Different Renewable Raw Materials. Fermentation 2018, 4, 36. [CrossRef]

4. Liu, T.; Huang, S.; Geng, A. Recombinant Diploid Saccharomyces cerevisiae Strain Development for Rapid Glucose and Xylose Co-Fermentation. Fermentation 2018, 4, 59. [CrossRef] 
5. Murdoch, I.; Powers, S.; Welch, A. Fluorinated Phenylalanine Precursor Resistance in Yeast. Fermentation 2018, 4, 41. [CrossRef]

6. Varelas, V.; Sotiropoulou, E.; Karambini, X.; Liouni, M.; Nerantzis, E. Impact of Glucose Concentration and $\mathrm{NaCl}$ Osmotic Stress on Yeast Cell Wall $\beta$-D-Glucan Formation during Anaerobic Fermentation Process. Fermentation 2017, 3, 44. [CrossRef]

7. Papagianni, M.; Papamichael, E. A Pichia anomala Strain (P. anomala M1) Isolated from Traditional Greek Sausage is an Effective Producer of Extracellular Lipolytic Enzyme in Submerged Fermentation. Fermentation 2017, 3, 43. [CrossRef]

8. Ravasio, D.; Carlin, S.; Boekhout, T.; Groenewald, M.; Vrhovsek, U.; Walther, A.; Wendland, J. Adding Flavor to Beverages with Non-Conventional Yeasts. Fermentation 2018, 4, 15. [CrossRef]

9. Long, D.; Wilkinson, K.; Taylor, D.; Jiranek, V. Novel Wine Yeast for Improved Utilisation of Proline during Fermentation. Fermentation 2018, 4, 10. [CrossRef]

10. Yang, F.; Heit, C.; Inglis, D. Cytosolic Redox Status of Wine Yeast (Saccharomyces cerevisiae) under Hyperosmotic Stress during Icewine Fermentation. Fermentation 2017, 3, 61. [CrossRef]

11. Ruíz-Muñoz, M.; Bernal-Grande, M.; Cordero-Bueso, G.; González, M.; Hughes-Herrera, D.; Cantoral, J. A Microtiter Plate Assay as a Reliable Method to Assure the Identification and Classification of the Veil-Forming Yeasts during Sherry Wines Ageing. Fermentation 2017, 3, 58. [CrossRef]

12. Barry, J.; Metz, M.; Hughey, J.; Quirk, A.; Bochman, M. Two Novel Strains of Torulaspora delbrueckii Isolated from the Honey Bee Microbiome and Their Use in Honey Fermentation. Fermentation 2018, 4, 22. [CrossRef]

13. Willaert, R. Micro- and Nanoscale Approaches in Antifungal Drug Discovery. Fermentation 2018, 4, 43. [CrossRef]

(C) 2018 by the author. Licensee MDPI, Basel, Switzerland. This article is an open access article distributed under the terms and conditions of the Creative Commons Attribution (CC BY) license (http:/ / creativecommons.org/licenses/by/4.0/). 